\title{
Nestor3D: multiple alignment of protein structures based on ligand or prosthetic group position Jean-Christophe Nebel*
}

\author{
Address: School of Computing \& Information Systems, Kingston University, Kingston-upon-Thames, Surrey, KT1 2EE, UK. \\ Email: Jean-Christophe Nebel* - J.Nebel@kingston.ac.uk \\ * Corresponding author
}

from BioSysBio: Bioinformatics and Systems Biology Conference

Edinburgh, UK, 14-15 July 2005

Published: 2I September 2005

BMC Bioinformatics 2005, 6(Suppl 3):S10

On one hand, high-throughput structural genomics projects deliver protein $3 \mathrm{D}$ structures regardless of the state of their functional annotation. On the other hand, the number of protein sequences still exceeds the number of 3D structures by more than 50 fold. Therefore, structure-based prediction of protein function and protein structure prediction are essential fields of research of the post-genomics era. These two challenges can be addressed by focusing on the most function-related parts of a protein structure, namely active sites. Nestor3D is a new software package, which allows the multiple alignment of protein structures based on structural elements involved in active site activity, i.e. ligands or prosthetic groups. According to our knowledge, no other package explores local atomiclevel similarity based on multiple comparisons. Nestor3D generates consensus 3D patterns including atom, chemical group and cavity positions. Depending on the set of structures used in the simultaneous alignment, 3D patterns can be used either as constraints for homology modelling, as template for drug design or for the detection of putative active sites. In addition, Nestor3D can also produce similarity matrices of proteins based on structural elements of active sites of interest. These matrices provide valuable information regarding how protein functions relate to each other. The system was validated using more than 200 protein chains representing proteins containing porphyrin rings. Examples suggest the generated 3D patterns are biologically meaningful, i.e. they are 3D motifs. Finally, comparisons with ClustalW indicate Nestor3D provides an added value to multiple sequence-based alignment methods. Further developments will include multiple alignments based on PROSITE patterns. Nestor3D can be downloaded for free from the following address: http://www.kingston.ac.uk/ ku33185/

Nestor3D.html 